\title{
ON THE CONE CONJECTURE FOR CALABI-YAU MANIFOLDS WITH PICARD NUMBER TWO
}

\author{
Vladimir Lazić And Thomas Peternell
}

\begin{abstract}
Following a recent work of Oguiso, we calculate explicitly the groups of automorphisms and birational automorphisms on a Calabi-Yau manifold with Picard number two. When the group of birational automorphisms is infinite, we prove that the Cone conjecture of Morrison and Kawamata holds.
\end{abstract}

\section{Contents}

1. Introduction

2. Preliminaries

3. Calculating $\operatorname{Aut}(X)$ and $\operatorname{Bir}(X)$

4. Structures of $\operatorname{Nef}(X)$ and $\overline{\operatorname{Mov}}(X)$

\section{Introduction}

The Cone conjecture of Morrison and Kawamata is concerned with the structure of the nef and the movable cones on a Calabi-Yau manifold in presence of automorphisms or birational automorphisms. To be more precise, consider a Calabi-Yau manifold $X$ with nef cone $\operatorname{Nef}(X)$, the movable cone $\overline{\operatorname{Mov}}(X)$, and effective cone $\operatorname{Eff}(X)$. A Calabi-Yau manifold in our context is a projective manifold $X$ with trivial canonical bundle, such that $H^{1}\left(X, \mathcal{O}_{X}\right)=0$. As usual, $\operatorname{Aut}(X)$, respectively $\operatorname{Bir}(X)$, denotes the group of automorphisms, respectively birational automorphisms of $X$. Then, the Cone conjecture can be stated as follows.

Conjecture 1.1. Let $X$ be a Calabi-Yau manifold.

(1) There exists a rational polyhedral cone $\Pi$ which is a fundamental domain for the action of $\operatorname{Aut}(X)$ on $\operatorname{Nef}(X) \cap \operatorname{Eff}(X)$, in the sense that

$$
\operatorname{Nef}(X) \cap \operatorname{Eff}(X)=\bigcup_{g \in \operatorname{Aut}(X)} g^{*} \Pi,
$$

and int $\Pi \cap \operatorname{int} g^{*} \Pi=\emptyset$ unless $g^{*}=\mathrm{id}$.

(2) There exists a rational polyhedral cone $\Pi^{\prime}$ which is a fundamental domain for the action of $\operatorname{Bir}(X)$ on $\overline{\operatorname{Mov}}(X) \cap \operatorname{Eff}(X)$.

Received by the editors February 25, 2013. 
There is also the following weaker form.

Conjecture 1.2. Let $X$ be a Calabi-Yau manifold.

(1) There exists a (not necessarily closed) cone $\Pi$ which is a weak fundamental domain for the action of $\operatorname{Aut}(X)$ on $\operatorname{Nef}(X) \cap \operatorname{Eff}(X)$, in the sense that

$$
\operatorname{Nef}(X) \cap \operatorname{Eff}(X)=\bigcup_{g \in \operatorname{Aut}(X)} g^{*} \Pi,
$$

int $\Pi \cap \operatorname{int} g^{*} \Pi=\emptyset$ unless $g^{*}=\mathrm{id}$, and for every $g \in \operatorname{Aut}(X)$, the intersection $\Pi \cap g^{*} \Pi$ is contained in a rational hyperplane.

(2) There exists a polyhedral cone $\Pi^{\prime}$ which is a weak fundamental domain for the action of $\operatorname{Bir}(X)$ on $\overline{\operatorname{Mov}}(X) \cap \operatorname{Eff}(X)$.

For the study of the Cone conjectures, the action

$$
r: \operatorname{Bir}(X) \rightarrow \operatorname{GL}\left(N^{1}(X)\right)
$$

on the Neron-Severi group $N^{1}(X)$ is important. We denote by $\mathcal{B}(X)$ its image, and by $\mathcal{A}(X)$ the image of the automorphism group.

Based on and inspired by recent work of Oguiso [6], we prove the following results.

Theorem 1.3. Let $X$ be a Calabi-Yau manifold of Picard number 2. Then, either $|\mathcal{A}(X)| \leq 2$, or $\mathcal{A}(X)$ is infinite; and either $|\mathcal{B}(X)| \leq 2$, or $\mathcal{B}(X)$ is infinite.

In fact, we explicitly calculate the groups $\mathcal{A}(X)$ and $\mathcal{B}(X)$, and for more detailed information we refer to Section 3. The consequences for the Cone conjectures can be summarized as follows.

Theorem 1.4. Let $X$ be a Calabi-Yau manifold with Picard number 2. Then

(1) If the group $\operatorname{Bir}(X)$ is finite, then the weak Cone conjecture holds on $X$.

(2) If the group $\operatorname{Bir}(X)$ is infinite, then the Cone conjecture holds on $X$.

Oguiso in [6] showed that there are indeed Calabi-Yau 3-folds $X$ with $\rho(X)=2$ and with infinite $\operatorname{Bir}(X)$, as well as hyperkähler 4-folds $X$ with $\rho(X)=2$ and with infinite $\operatorname{Aut}(X)$.

\section{Preliminaries}

In this section, we give some basic definitions and gather results which we need in this paper.

A Calabi-Yau manifold of dimension $n$ is a projective manifold $X$ with trivial canonical bundle $K_{X} \simeq \mathcal{O}_{X}$ such that $H^{1}\left(X, \mathcal{O}_{X}\right)=0$. In particular, we do not require $X$ to be simply connected.

Let $N^{1}(X)$ be the Neron-Severi group, generated by the classes of the line bundles on $X$ and let $N^{1}(X)_{\mathbb{R}}$ be the corresponding real vector space in $H^{2}(X, \mathbb{R})$. As usual, $\operatorname{Nef}(X) \subseteq N^{1}(X)_{\mathbb{R}}$ denotes the closed cone of nef divisors, $\operatorname{Big}(X)$ stands for the open cone of big divisors, $\overline{\operatorname{Mov}}(X)$ is the closure of the cone generated by mobile divisors (that is, effective divisors whose base locus does not contain divisors), and $\operatorname{Mov}(X)$ is its interior. Finally, $\operatorname{Eff}(X)$ is the effective cone, and $\overline{\operatorname{Eff}}(X)$ is the pseudo-effective cone (the closure of the effective cone, or equivalently, the closure of the big cone). 
On a normal $\mathbb{Q}$-factorial projective variety $X$ with terminal singularities and nef canonical class, $\operatorname{Aut}(X)$ denotes the automorphism group and $\operatorname{Bir}(X)$ the group of birational automorphisms. We obtain a natural homomorphism

$$
r: \operatorname{Bir}(X) \rightarrow \operatorname{GL}\left(N^{1}(X)\right)
$$

given by $g \mapsto g^{*}$.

Notation 2.1. Assume that a Calabi-Yau manifold $X$ has Picard number $\rho(X)=2$. We let $\ell_{1}, \ell_{2}$ be the two boundary rays of $\operatorname{Nef}(X)$, and let $m_{1}, m_{2}$ be the boundary rays of $\overline{\operatorname{Mov}}(X)$. We fix non-trivial elements $x_{i} \in \ell_{i}$ and $y_{i} \in m_{i}$. We set

$$
\mathcal{A}(X)=r(\operatorname{Aut}(X)) \text { and } \mathcal{B}(X)=r(\operatorname{Bir}(X)) .
$$

It is well-known, see for instance [6, Proposition 2.4], that the $\operatorname{group} \operatorname{Bir}(X)$ is finite if and only if $\mathcal{B}(X)$ is, and similarly for $\operatorname{Aut}(X)$ and $\mathcal{A}(X)$.

Recall also the following result [6, Proposition 3.1].

Proposition 2.2. Let $X$ be a Calabi-Yau manifold of dimension $n$ such that $\rho(X)=2$.

(1) If $n$ is odd, or if one of the $\ell_{i}$ is rational, then every non-trivial element of $\mathcal{A}(X)$ has order 2 .

(2) If one of the $m_{i}$ is rational, then every non-trivial element of $\mathcal{B}(X)$ has order 2 .

As a consequence, by using Burnside's theorem, Oguiso obtains:

Theorem 2.3. Let $X$ be a Calabi-Yau manifold of dimension $n$ such that $\rho(X)=2$.

(1) If $n$ is odd, then $\operatorname{Aut}(X)$ is finite.

(2) If $n$ is even and one of the rays $\ell_{i}$ is rational, then $\operatorname{Aut}(X)$ is finite.

(3) If one of the rays $m_{i}$ is rational, then $\operatorname{Bir}(X)$ is finite.

Proposition 3.3 below makes this result more precise. In contrast to Theorem 2.3, Oguiso constructed an example of Calabi-Yau manifold with $\rho(X)=2$ such that $\operatorname{Bir}(X)$ is infinite. In this example both rays $m_{i}$ are irrational, and we recall it in Example 4.6 .

If $g$ is any element of $\mathcal{B}(X)$, then $\operatorname{det} g= \pm 1$ since $g$ acts on the integral lattice $N^{1}(X)$. We introduce the notations

$$
\mathcal{A}^{+}(X)=\{g \in \mathcal{A}(X) \mid \operatorname{det} g=1\}
$$

and

$$
\mathcal{A}^{-}(X)=\{g \in \mathcal{A}(X) \mid \operatorname{det} g=-1\} ;
$$

and similarly $\mathcal{B}^{+}(X)$ and $\mathcal{B}^{-}(X)$. Note that each $g \in \mathcal{A}(X)$ restricts to an action on the set $\ell_{1} \cup \ell_{2}$, and each $g \in \mathcal{B}(X)$ restricts to an action on the set $m_{1} \cup m_{2}$. Moreover, since the cone $\overline{\mathrm{Eff}}(X)$ does not contain lines, this "restricted" action completely determines $g$. Additionally, each $g \in \mathcal{A}(X)$ is completely determined by $g x_{1}$ since det $g= \pm 1$. Similarly, each $g \in \mathcal{B}(X)$ is completely determined by $g y_{1}$.

We frequently and without explicit mention use the following well-known lemma, see for instance [4, Lemma 1.5].

Lemma 2.4. Let $X$ be a Calabi-Yau manifold. Then, $g \in \operatorname{Bir}(X)$ is an automorphism if and only if there exists an ample divisor $H$ on $X$ such that $g^{*} H$ is ample. 
We also use the following result [3, Theorem 5.7], [4, Corollary 2.7], [2, Theorem 3.8].

Theorem 2.5. Let $X$ be a Calabi-Yau manifold. Then, the cones $\operatorname{Nef}(X)$ and $\overline{\operatorname{Mov}}(X)$ are locally rational polyhedral in $\operatorname{Big}(X)$.

\section{Calculating $\operatorname{Aut}(X)$ and $\operatorname{Bir}(X)$}

In this section we calculate explicitly the groups $\mathcal{A}(X)$ and $\mathcal{B}(X)$ on a Calabi-Yau manifold with Picard number 2 . We start with some elementary observations.

Lemma 3.1. Let $X$ be a Calabi-Yau manifold such that $\rho(X)=2$. If $g \in \mathcal{B}^{-}(X)$, then $g^{2}=\mathrm{id}$.

Proof. By assumption there exist $\alpha>0$ and $\beta>0$ such that $g y_{1}=\alpha y_{2}$ and $g y_{2}=\beta y_{1}$. However, then $g^{2} y_{1}=\alpha \beta y_{1}$ and $g^{2} y_{2}=\alpha \beta y_{2}$, and we have $g^{2} \in \mathcal{A}^{+}(X)$. Therefore $\operatorname{det}\left(g^{2}\right)=(\alpha \beta)^{2}=1$, so $\alpha \beta=1$. Thus, $g^{2}$ is the identity.

Lemma 3.2. Let $X$ be a Calabi-Yau manifold such that $\rho(X)=2$. Then, $\mathcal{B}^{-}(X)=$ $\mathcal{B}^{+}(X) g$ for any $g \in \mathcal{B}^{-}(X)$. Similarly, $\mathcal{A}^{-}(X)=\mathcal{A}^{+}(X) h$ for any $h \in \mathcal{A}^{-}(X)$.

In particular, if $\mathcal{B}(X)$ is infinite, so is $\mathcal{B}^{+}(X)$; and if $\mathcal{A}(X)$ is infinite, so is $\mathcal{A}^{+}(X)$.

Proof. Let $g, g^{\prime} \in \mathcal{B}^{-}(X)$. Then, $g^{\prime} g=f \in \mathcal{B}^{+}(X)$, and since $g^{2}=$ id by Proposition 2.2, we have $g^{\prime}=f g \in \mathcal{B}^{+}(X) g$. The proof in the case of automorphisms is identical.

Proposition 3.3. Let $X$ be a Calabi-Yau manifold such that $\rho(X)=2$. If $\mathcal{A}(X)$ is finite, then $\left|\mathcal{A}^{+}(X)\right|=1$ and $|\mathcal{A}(X)| \leq 2$. If $\mathcal{B}(X)$ is finite, then $\left|\mathcal{B}^{+}(X)\right|=1$ and $|\mathcal{B}(X)| \leq 2$.

In particular, if $n$ is odd, or if one of the $\ell_{i}$ is rational, then $|\mathcal{A}(X)| \leq 2$.

Proof. Assume that $\mathcal{A}(X)$ is finite, and fix $g \in \mathcal{A}(X)$. If $g \in \mathcal{A}^{+}(X)$, then there exists $\alpha>0$ such that $g x_{1}=\alpha x_{1}$. Then, $g^{m}=$ id for some positive integer $m$, hence $\alpha^{m}=1$, and therefore $\alpha=1$ and $\mathcal{A}^{+}(X)=\{$ id $\}$. Now $|\mathcal{A}(X)| \leq 2$ by Lemma 3.2. The proof for $\mathcal{B}(X)$ is the same, and the last claim follows from Theorem 2.3.

Proposition 3.3 can also be directly deduced from the following elementary lemma, simplifying calculations in [6].

Lemma 3.4. Let $X$ be an $n$-dimensional Calabi-Yau manifold with $\rho(X)=2$. Assume that $\left|\mathcal{A}^{+}(X)\right| \neq 1$. Then,

$$
x_{1}^{m} \cdot x_{2}^{n-m}=0
$$

for all $m$ unless $n=2 m$.

If $n=2 m$, then $x_{1}^{m} \neq 0$ and $x_{2}^{m} \neq 0$.

Proof. Let $f$ be a non-trivial element in $\mathcal{A}^{+}$. Then, $f x_{1}=\alpha x_{1}$ and $f x_{2}=\alpha^{-1} x_{2}$ with $\alpha>0, \alpha \neq 1$. Then

$$
\left(f x_{1}\right)^{m} \cdot\left(f x_{2}\right)^{n-m}=\alpha^{2 m-n} x_{1}^{m} \cdot x_{2}^{n-m} .
$$

On the other hand,

$$
\left(f x_{1}\right)^{m} \cdot\left(f x_{2}\right)^{n-m}=x_{1}^{m} \cdot x_{2}^{n-m},
$$

hence $x_{1}^{m} \cdot x_{2}^{n-m}=0$ unless $n=2 m$. 
For the second statement, observe that $x_{1}+x_{2}$ is an ample class, hence

$$
0<\left(x_{1}+x_{2}\right)^{n}=\left(\begin{array}{c}
n \\
m
\end{array}\right) x_{1}^{m} \cdot x_{2}^{m},
$$

and therefore the classes $x_{i}^{m}$ are non-zero.

Corollary 3.5. Let $X$ be a Calabi-Yau manifold of dimension $n$ such that $\rho(X)=2$. If the group $\operatorname{Aut}(X)$ is infinite, then the following holds.

(1) $n$ is even and the rays $\ell_{i}$ are irrational.

(2) $\operatorname{Nef}(X)=\overline{\operatorname{Eff}}(X)$, and $\operatorname{Nef}(X) \cap \operatorname{Eff}(X)=\operatorname{Amp}(X)$.

(3) $c_{n-1}(X)=0$ in $H^{2 n-2}(X, \mathbb{Q})$.

Proof. Claim (1) is Oguiso's Theorem 2.3.

For the first part of $(2)$, if $\operatorname{Nef}(X) \neq \overline{\operatorname{Eff}}(X)$, then at least one boundary ray of $\operatorname{Nef}(X)$ is rational by Theorem 2.5. This contradicts (1). For the second part of (2), without loss of generality it suffices to show that $x_{1}$ is not effective. Otherwise, we can write $x_{1}=\sum \delta_{j} D_{j} \geq 0$ as a sum of at least two prime divisors, since $x_{1}$ is irrational. However, then $\ell_{1}$ is not an extremal ray of the cone $\operatorname{Nef}(X)=\overline{\operatorname{Eff}}(X)$, a contradiction.

For (3), note that $\left|\mathcal{A}^{+}(X)\right| \geq 2$ by Lemma 3.2. Pick a non-trivial element $f \in \mathcal{A}^{+}(X)$, and let $\alpha \neq 1$ be a positive number such that $f x_{1}=\alpha x_{1}$. Then,

$$
\alpha x_{1} \cdot c_{n-1}(X)=f x_{1} \cdot c_{n-1}(X)=x_{1} \cdot c_{n-1}(X)
$$

since the Chern class $c_{n-1}(X)$ is invariant under $f$. Thus, $x_{1} \cdot c_{n-1}(X)=0$; similarly we get $x_{2} \cdot c_{n-1}(X)=0$. Therefore $c_{n-1}(X)=0$ as $\left\{x_{1}, x_{2}\right\}$ is a basis of $N_{\mathbb{R}}^{1}(X)$.

Remark 3.6. (1) The same arguments as in Corollary 3.5 yield

$$
c_{i_{1}}(X) \cdot \ldots \cdot c_{i_{r}}(X)=0
$$

if $i_{1}+\cdots+i_{r}=n-1$.

(2) We do not know of any example of a simply connected Calabi-Yau manifold $X$ in the strong sense, (i.e., such that $H^{q}\left(X, \mathcal{O}_{X}\right)=0$ for $1 \leq q \leq n-1$ ) of even dimension $n$ such that $c_{n-1}(X)=0$. One might wonder whether any simply connected irreducible projective manifold $X$ of dimension $n$ with $\omega_{X} \simeq \mathcal{O}_{X}$ and $c_{n-1}(X)=0$ is a hyperkähler manifold.

In some further cases, the even dimensional case can be treated.

Theorem 3.7. Let $X$ be a Calabi-Yau manifold of even dimension $n$. If $\rho(X)=2$ and if $c_{2}(X)$ can be represented by a positive closed $(2,2)$-form, then $\operatorname{Aut}(X)$ is finite.

Proof. Arguing by contradiction, we suppose that there is an automorphism $f \in \mathcal{A}^{+}(X)$ of infinite order; cf. Lemma 3.2. Write $n=2 m$. Then, $x_{1}^{m} \neq 0$ and $x_{2}^{m} \neq 0$ by Lemma 3.4 .

Suppose that $m$ is even, and write $m=2 k$. Then

$$
x_{1}^{2 k} \cdot c_{2}(X)^{k}>0
$$

by our positivity assumption on $c_{2}(X)$. On the other hand,

$$
x_{1}^{2 k} \cdot c_{2}(X)^{k}=\left(f x_{1}\right)^{2 k} \cdot c_{2}(X)^{k}=\alpha^{2 k} x_{1}^{2 k} \cdot c_{2}(X)^{k}
$$

since $c_{2}(X)$ is invariant under $f$. Since $\alpha \neq 1$, this is a contradiction. 
If $m$ is odd, we write $n=4 s+2$ and argue with $x_{1}^{2 s} \cdot c_{2}(X)^{s+1}$.

Notice that for every projective manifold $X$ of dimension $n$ with nef canonical bundle, the second Chern class $c_{2}(X)$ has the following positivity property (Miyaoka [5]):

$$
c_{2}(X) \cdot H_{1} \cdot \ldots \cdot H_{n-2} \geq 0
$$

for all ample line bundles $H_{j}$.

Concerning bounds for $\mathcal{B}(X)$, we have:

Proposition 3.8. Let $X$ be a Calabi-Yau manifold such that $\rho(X)=2$. Assume that $\operatorname{Nef}(X) \nsubseteq \operatorname{Mov}(X)$. Then, $\mathcal{A}^{+}(X)=\mathcal{B}^{+}(X)$. In particular, if the dimension of $X$ is odd, then $|\mathcal{B}(X)| \leq 2$.

Proof. The condition $\operatorname{Nef}(X) \nsubseteq \operatorname{Mov}(X)$ implies that one of the rays $\ell_{i}$ is an extremal ray of $\overline{\operatorname{Mov}}(X)$. Hence, without loss of generality, we may assume that $m_{1}=\ell_{1}$. Let $g$ be a non-trivial element of $\mathcal{B}^{+}(X)$. Then, $g \ell_{1}=g m_{1}=m_{1}$, and $m_{1}$ is an extremal ray of the cone

$$
\mathbb{R}_{+} m_{1}+\mathbb{R}_{+} g \ell_{2}=\mathbb{R}_{+} g \ell_{1}+\mathbb{R}_{+} g \ell_{2}=g \operatorname{Nef}(X) .
$$

This implies that $g \operatorname{Nef}(X)$ intersects the interior of $\operatorname{Nef}(X)$, and hence $g \in \mathcal{A}(X)$ by Lemma 2.4. This proves the first claim.

The second claim then follows from Proposition 3.3.

Theorem 3.9. Let $X$ be a Calabi-Yau manifold such that $\rho(X)=2$. Then, either $\left|\mathcal{A}^{+}(X)\right|=1$ or $\mathcal{A}^{+}(X) \simeq \mathbb{Z}$; and either $\left|\mathcal{B}^{+}(X)\right|=1$ or $\mathcal{B}^{+}(X) \simeq \mathbb{Z}$.

Proof. Assume that $\left|\mathcal{A}^{+}(X)\right| \geq 2$. For every $g \in \mathcal{A}^{+}(X)$, let $\alpha_{g}$ be the positive number such that $g y_{1}=\alpha_{g} y_{1}$, and set

$$
\mathcal{S}=\left\{\alpha_{g} \mid g \in \mathcal{A}^{+}(X)\right\} .
$$

Note that $\mathcal{S}$ is a multiplicative subgroup of $\mathbb{R}^{*}$ and that the map

$$
\mathcal{A}^{+}(X) \rightarrow \mathcal{S}, \quad g \mapsto \alpha_{g}
$$

is an isomorphism of groups. We need to show that $\mathcal{S}$ is an infinite cyclic group.

We first show that $\mathcal{S}$ is, as a set, bounded away from 1 . Otherwise, we can pick a sequence $\left(g_{i}\right)$ in $\mathcal{A}^{+}(X)$ such that $\alpha_{g_{i}}$ converges to 1 . Fix two integral linearly independent classes $h_{1}$ and $h_{2}$ in $N^{1}(X)_{\mathbb{R}}$. Then, $g_{i} h_{1}$ converge to $h_{1}$ and $g_{i} h_{2}$ converge to $h_{2}$. Since $g_{i} h_{1}$ and $g_{i} h_{2}$ are also integral classes and $N^{1}(X)$ is a lattice in $N^{1}(X)_{\mathbb{R}}$; this implies that $g_{i} h_{1}=h_{1}$ and $g_{i} h_{2}=h_{2}$ for $i \gg 0$, and hence $g_{i}=$ id for $i \gg 0$.

Hence, the set $\mathcal{S}^{\prime}=\{\ln \alpha \mid \alpha \in \mathcal{S}\}$ is an additive subgroup of $\mathbb{R}$ which is discrete as a set. Then, it is a standard fact that $\mathcal{S}^{\prime}$, and hence $\mathcal{S}$ is isomorphic to $\mathbb{Z}$, cf. [1, 21.1].

The proof for the birational automorphism group is the same.

\section{Structures of $\operatorname{Nef}(X)$ and $\overline{\operatorname{Mov}}(X)$}

Proposition 4.1. Let $X$ be a Calabi-Yau manifold such that $\rho(X)=2$. If $\mathcal{A}(X)$ is finite, then the weak Cone conjecture holds for $\operatorname{Nef}(X)$. If $\mathcal{B}(X)$ is finite, then the weak Cone conjecture holds for $\overline{\operatorname{Mov}}(X)$. 
Proof. We only prove the statement about the nef cone, since the other statement is analogous. By Proposition 3.3, we have $|\mathcal{A}(X)| \leq 2$, hence we may assume that $|\mathcal{A}(X)|=2$. Fix an integral class $x \in \operatorname{Nef}(X)$, let $g \in \mathcal{A}^{-}(X)$, and consider the class $y=x+g x \in \operatorname{Nef}(X)$. Then, $y$ is fixed under the action of $\mathcal{A}(X)$. Since $g$ acts on $N^{1}(X)$, both $g x$ and $y$ must be integral. It is then obvious that $\Pi=\ell_{1}+\mathbb{R}_{+} y$ is a fundamental domain for the action of $\mathcal{A}(X)$ on $\operatorname{Nef}(X)$.

Remark 4.2. If $X$ is a Calabi-Yau manifold of odd dimension such that $\rho(X)=2$ and $\operatorname{Nef}(X) \nsubseteq \operatorname{Mov}(X)$, then the weak Cone conjecture holds for $\overline{\operatorname{Mov}}(X)$. The proof is analogous to that of Proposition 4.1, using Proposition 3.8.

Proposition 4.3. Let $X$ be a Calabi-Yau manifold such that $\rho(X)=2$. Assume that $\operatorname{Nef}(X) \subseteq \operatorname{Mov}(X)$. Then, the Cone conjecture holds for $\operatorname{Nef}(X)$.

Proof. By assumption, we have $\operatorname{Nef}(X) \subseteq \operatorname{Big}(X)$, and hence, the nef cone is rational polyhedral by Theorem 2.5. Then, argue as in the proof of Proposition 4.1.

Lemma 4.4. Let $X$ be a Calabi-Yau manifold with $\rho(X)=2$. Assume that $\operatorname{Bir}(X)$ is infinite. Then, $\overline{\operatorname{Mov}}(X) \cap \operatorname{Eff}(X)=\operatorname{Mov}(X)$.

Proof. The rays of $\overline{\operatorname{Mov}}(X)$ are irrational by Proposition 2.2, and therefore $\overline{\operatorname{Mov}}(X)=$ $\overline{\mathrm{Eff}}(X)$ by Theorem 2.5. We cannot have $y_{1} \in \operatorname{Eff}(X)$ : otherwise, we can write $y_{1}=$ $\sum \delta_{i} D_{i} \geq 0$ as a sum of at least two different prime divisors, since $m_{1}$ is irrational. However, then $m_{1}$ is not an extremal ray of the cone $\overline{\operatorname{Mov}}(X)=\overline{\operatorname{Eff}}(X)$, a contradiction. This concludes the proof.

Theorem 4.5. Let $X$ be a Calabi-Yau manifold with $\rho(X)=2$. If the group $\operatorname{Bir}(X)$ is infinite, then the Cone conjecture holds on $X$.

Proof. (i) First we show that the Cone conjecture holds for $\operatorname{Nef}(X)$ in case $\operatorname{Aut}(X)$ is infinite.

Note that $\operatorname{Nef}(X)=\overline{\operatorname{Eff}}(X)$ and $\operatorname{Nef}(X) \cap \operatorname{Eff}(X)=\operatorname{Amp}(X)$ by Corollary 3.5(2), and in particular we have $\mathcal{A}(X)=\mathcal{B}(X)$. By Lemma 3.2 and Theorem 3.9, we know that $\mathcal{A}(X)=\mathcal{A}^{+}(X) \cup \mathcal{A}^{-}(X)$, where $\mathcal{A}^{+}(X) \simeq \mathbb{Z}$ and $\mathcal{A}^{-}(X)=\mathcal{A}^{+}(X) g$ for any $g \in \mathcal{A}^{-}(X)$.

Assume first that $\mathcal{A}(X)=\mathcal{A}^{+}(X) \simeq \mathbb{Z}$. Let $h$ be a generator of $\mathcal{A}(X)$, let $x$ be any point in $\operatorname{Amp}(X)$, and denote

$$
\Pi=\mathbb{R}_{+} x+\mathbb{R}_{+} h x .
$$

It is then straightforward to check that $\Pi$ is a fundamental domain for the action of $\mathcal{A}(X)$ on $\operatorname{Amp}(X)$. Indeed, it is clear that the cones $h^{k} \Pi$ have disjoint interiors, and to see that they cover $\operatorname{Amp}(X)$, it suffices to notice that the rays $\mathbb{R}_{+} h^{k} x$ converge to $\ell_{1}, \ell_{2}$, when $k \rightarrow \pm \infty$.

Now assume that $\mathcal{A}^{-}(X) \neq \emptyset$. Let $f$ be a generator of $\mathcal{A}^{+}(X)$, let $\tau$ be an element of $\mathcal{A}^{-}(X)$, and let $x$ be an integral class in $\operatorname{Amp}(X)$. Set

$$
z_{1}=x+\tau x \quad \text { and } \quad z_{2}=z_{1}+f z_{1},
$$

and note that $z_{1}$ and $z_{2}$ are integral classes since $\tau$ and $f$ act on $N^{1}(X)$. Denote $\theta=f \tau \in \mathcal{A}^{-}(X)$. Then, $\tau^{2}=\theta^{2}=$ id by Lemma 3.1, and hence

$$
\theta \tau=(f \tau) \tau=f \quad \text { and } \quad \theta f=\theta(\theta \tau)=\tau .
$$


This implies

$$
\tau z_{1}=z_{1}, \quad \theta z_{1}=f z_{1}, \quad \theta z_{2}=z_{2}
$$

Now, let

$$
\Pi=\mathbb{R}_{+} z_{1}+\mathbb{R}_{+} z_{2} .
$$

Then, $\Pi$ is a rational polyhedral cone, and we claim that $\Pi$ is a fundamental domain for the action of $\mathcal{A}(X)$ on $\operatorname{Amp}(X)$.

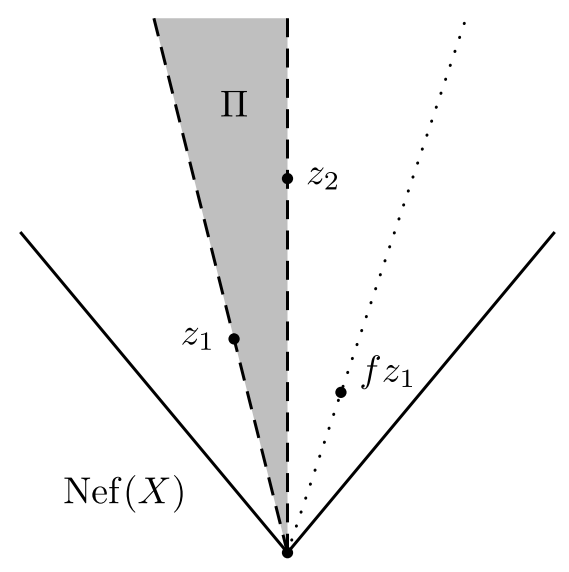

First, by (4.1) we have

$$
\theta \Pi=\mathbb{R}_{+} \theta z_{1}+\mathbb{R}_{+} \theta z_{2}=\mathbb{R}_{+} f z_{1}+\mathbb{R}_{+} z_{2},
$$

and thus

$$
\Pi \cup \theta \Pi=\mathbb{R}_{+} z_{1}+\mathbb{R}_{+} f z_{1}
$$

This implies

$$
\bigcup_{k \in \mathbb{Z}} f^{k}(\Pi \cup \theta \Pi)=\operatorname{Amp}(X)
$$

as in the first part of the proof, and therefore,

$$
\bigcup_{g \in \mathcal{A}(X)} g \Pi=\operatorname{Amp}(X) .
$$

Second, assume that there exists $\lambda \in \mathcal{A}(X)$ such that int $\Pi \cap \operatorname{int} \lambda \Pi \neq \emptyset$. Then, possibly after replacing $\lambda$ by $\lambda^{-1}$, this implies that $\lambda z_{1} \subseteq$ int $\Pi$ or $\lambda z_{2} \subseteq$ int $\Pi$. If $\lambda z_{1} \subseteq$ int $\Pi$, then by Lemma 3.2 there exists $k \in \mathbb{Z}$ such that $\lambda=f^{k} \tau$, hence $\lambda z_{1}=f^{k} z_{1} \in \operatorname{int} \Pi$ by (4.1), which is clearly impossible. Similarly, if $\lambda z_{2} \subseteq$ int $\Pi$, again by Lemma 3.2 there exists $\ell \in \mathbb{Z}$ such that $\lambda=f^{\ell} \theta$, hence $\lambda z_{2}=f^{\ell} z_{2} \in \operatorname{int} \Pi$ by (4.1), a contradiction. This finishes the proof of (i).

(ii) Next, we show that the Cone conjecture holds for $\operatorname{Nef}(X)$ if $\operatorname{Aut}(X)$ is finite, but $\operatorname{Bir}(X)$ is infinite. Here, $\operatorname{Nef}(X) \subseteq \operatorname{Mov}(X)$ by Lemma 3.2 and Proposition 3.8. Then, the Cone conjecture for $\operatorname{Nef}(X)$ holds by Proposition 4.3.

(iii) Finally, note that $\overline{\operatorname{Mov}}(X) \cap \operatorname{Eff}(X)=\operatorname{Mov}(X)$ by Lemma 4.4; hence the proof of the Cone conjecture for $\operatorname{Mov}(X)$ is the same as that of (i) by a simple adaption. 
Example 4.6. We recall [6, Proposition 6.1]. Oguiso constructs a Calabi-Yau 3-fold $X$ with Picard number 2, obtained as the intersection of general hypersurfaces in $\mathbb{P}^{3} \times \mathbb{P}^{3}$ of bidegrees $(1,1),(1,1)$, and $(2,2)$, which has the following properties: $x_{1}$ and $x_{2}$ are rational, $y_{1}=(3+2 \sqrt{2}) x_{2}-x_{1}, y_{2}=(3+2 \sqrt{2}) x_{1}-x_{2}$, there are two birational involutions $\tau_{1}$ and $\tau_{2}$ such that $\tau_{1} \tau_{2}$ is of infinite order, and the group $\operatorname{Bir}(X)$ is generated by $\operatorname{Aut}(X)$ and by $\tau_{1}$ and $\tau_{2}$.

We now show that Example 4.6 is a typical example of a Calabi-Yau manifold with Picard number 2 and with infinite group of birational automorphisms.

Theorem 4.7. Let $X$ be a Calabi-Yau manifold of dimension $n$ and with $\rho(X)=2$. Assume that $\operatorname{Bir}(X)$ is infinite.

(1) Let $f$ be a generator of $\mathcal{B}^{+}(X)$, and let $\alpha>0$ be the real number such that $f y_{1}=\alpha y_{1}$. Then, $[\mathbb{Q}(\alpha): \mathbb{Q}]=2$.

(2) Let $\{v, w\}$ be any integral basis of $N^{1}(X)_{\mathbb{R}}$. Then, $m_{1}=\mathbb{R}_{+}(a v+b w)$ and $m_{2}=$ $\mathbb{R}_{+}(c v+d w)$, where $a, b, c, d \in \mathbb{Q}(\alpha)$.

(3) There exists a birational automorphism $\tau$ (possibly the identity) such that $\tau^{2} \in$ $\operatorname{Aut}(X)$, and a birational automorphism of infinite order $\sigma$ such that the group $\operatorname{Bir}(X)$ is generated by $\operatorname{Aut}(X)$ and by $\tau$ and $\sigma$.

Proof. By rescaling $y_{1}$ and $y_{2}$, we can assume that

$$
h=y_{1}+y_{2}
$$

is a primitive integral class in $N^{1}(X)_{\mathbb{R}}$. Denote

$$
h^{\prime}=f h=\alpha y_{1}+\frac{1}{\alpha} y_{2} \quad \text { and } \quad h^{\prime \prime}=f^{2} h=\alpha^{2} y_{1}+\frac{1}{\alpha^{2}} y_{2} ;
$$

these are again primitive integral classes since $\mathcal{B}(X)$ preserves $N^{1}(X)$. Then, an easy calculation shows that

$$
h+h^{\prime \prime}=\frac{\alpha^{2}+1}{\alpha} h^{\prime}
$$

and hence the number $\frac{\alpha^{2}+1}{\alpha}=\alpha+\frac{1}{\alpha}$ is an integer. Since

$$
y_{1}=\frac{1}{\alpha^{2}-1}\left(\alpha h^{\prime}-h\right),
$$

and $y_{1}$ is not rational by Theorem 2.3 , the number $\alpha$ cannot be rational, and (1) follows.

For (2) fix an integral basis $\{v, w\}$ of $N^{1}(X)_{\mathbb{R}}$, and write

$$
y_{1}=a v+b w \text { and } y_{2}=c v+d w .
$$

Then,

$$
h=(a+c) v+(b+d) w \quad \text { and } \quad h^{\prime}=(\alpha a+c / \alpha) v+(\alpha b+d / \alpha) w .
$$

Write $p=a+c$ and $q=\alpha a+c / \alpha$, and note that $p, q \in \mathbb{Z}$. Then, an easy calculation shows that $a, c \in \mathbb{Q}(\alpha)$, and similarly for $b$ and $d$.

Finally, for (3), note that by Theorem 3.9 and Lemma 3.2 , we have $\mathcal{B}(X)=$ $\mathcal{B}^{+}(X) \cup \mathcal{B}^{-}(X)$, where $\mathcal{B}^{+}(X)$ is infinite cyclic with generator $\sigma^{\prime}$, and $\mathcal{B}^{-}(X)=$ $\mathcal{B}^{+}(X) \tau^{\prime}$ for any $\tau^{\prime} \in \mathcal{B}^{-}(X)$. Pick $\tau, \sigma \in \operatorname{Bir}(X)$ such that

$$
r(\tau)=\tau^{\prime} \text { and } r(\sigma)=\sigma^{\prime}
$$


see Notation 2.1. Since $r\left(\tau^{2}\right)=\tau^{\prime 2}=$ id by Lemma 3.1, it follows that $\tau^{2}$ is an isomorphism by [6, Proposition 2.4]. Now if $\theta$ is any element of $\operatorname{Bir}(X)$, then there exist integers $k$ and $\ell$ such that $r(\theta)=\sigma^{k} \tau^{\prime \ell}=r\left(\sigma^{k} \tau^{\ell}\right)$, and we conclude again by [6, Proposition 2.4].

Remark 4.8. We are indebted to the referee for pointing out the following example, which provides a variety satisfying the assumptions of Theorem 4.7 in any dimension $n \geq 3$.

Let $X$ be the complete intersection

$$
H_{1} \cap H_{2} \cap \cdots \cap H_{n-1} \cap Q \subseteq \mathbb{P}^{n} \times \mathbb{P}^{n},
$$

where $n \geq 3$, where $H_{i}$ are general hypersurfaces of bidegree $(1,1)$, and where $Q$ is a general hypersurface of bidegree $(2,2)$. Then, $X$ is a simply connected Calabi-Yau $n$-fold with Picard number two. More precisely, $\operatorname{Pic}(X)=\mathbb{Z} L_{1} \oplus \mathbb{Z} L_{2}$, where $L_{1}$ and $L_{2}$ are pullbacks of the hyperplane classes of factors $\mathbb{P}^{n}$. Consider the two birational involutions $\iota_{1}, \iota_{2}$ induced by the two natural projections of $X$ to $\mathbb{P}^{n}$. Then, $\iota_{1} \iota_{2}$ is a birational automorphism of $X$ of infinite order. The last statement can be checked by computing $\left(\iota_{1} \iota_{2}\right)^{*} L_{i}$ as in [6, Proposition 6.1].

Remark 4.9. One can obtain a similar description of the cone $\operatorname{Nef}(X)$ when the automorphism group of $X$ is infinite.

Basically there are two types of simply connected irreducible Calabi-Yau manifolds: those which do not carry any holomorphic forms of intermediate degree - these manifolds are often simply called Calabi-Yau manifolds - and hyperkähler manifolds carrying a non-degenerate holomorphic 2-form. While in the hyperkähler case the nef cone can be irrational by [6, Proposition 1.3], it is believed that the nef cone of a "strict" Calabi-Yau manifold with, say, $\rho(X)=2$, must be rational. The evidence is provided by the fact that in odd dimensions $\operatorname{Aut}(X)$ is finite, and then the Cone conjecture would imply the rationality. In even dimensions, we saw that an infinite automorphism group on a strict Calabi-Yau manifold with Picard number two is possible only in very special circumstances.

\section{Acknowledgments}

We thank J. Hausen, A. Prendergast-Smith, D.-Q. Zhang and the referee for very useful comments and suggestions. We were supported by the DFG-Forschergruppe 790 "Classification of Algebraic Surfaces and Compact Complex Manifolds".

\section{References}

[1] O. Forster, Lectures on Riemann surfaces, Graduate Texts in Mathematics, 81, Springer-Verlag, New York, 1981.

[2] A.-S. Kaloghiros, A. Küronya and V. Lazić, Finite generation and geography of models (to appear in "Minimal models and extremal rays", Adv. Stud. Pure Math., Math. Soc. Japan, Tokyo (2012), arXiv:1202.1164).

[3] Y. Kawamata, Crepant blowing-up of 3-dimensional canonical singularities and its application to degenerations of surfaces, Ann. of Math. (2) 127(1) (1988), 93-163.

[4] - On the cone of divisors of Calabi-Yau fiber spaces, Internat. J. Math. 8 (1997), 665-687.

[5] Y. Miyaoka, The Chern classes and Kodaira dimension of a minimal variety, in Algebraic Geometry, Sendai, 1985, Vol. 10 of Adv. Stud. Pure Math., 449-476, North Holland, Amsterdam (1987). 
[6] K. Oguiso, Automorphism groups of Calabi-Yau manifolds of Picard number two (to appear in J. Algebraic Geom. (2012), arXiv:1206.1649).

Mathematisches Institut, Universität Bonn, Endenicher Allee 60, 53115 Bonn, Germany E-mail address: lazic@math.uni-bonn.de

Mathematisches Institut, Universität Bayreuth, 95440 Bayreuth, Germany

E-mail address: thomas.peternell@uni-bayreuth.de 
